\title{
Association between birth weight and adult blood pressure in twins: historical cohort study
}

\author{
N R Poulter, C L Chang, A J MacGregor, H Snieder, T D Spector
}

Editorial by Leon
Cardiovascular
Studies Unit,
Department of
Clinical
Pharmacology,
Imperial College
School of Medicine,
St Mary's Campus,
London W2 1PG
N R Poulter
director
C L Chang
research fellow
Twin Research and
Genetic
Epidemiology Unit,
St Thomas's
Hospital, Guy's and
St Thomas's
Hospital Trust,
London SE1 7EH
A J MacGregor
Arthritis and
Rheumatism
Campaign senior
fellow
H Snieder
genetic epidemiologist
T D Spector
director
Correspondence to:
N R Poulter
npolter@icacut

n.poulter@ic.ac.uk

BMJ 1999;319:1330-3

website

extra

Extended versions of the tables

appear on the

BMJ's website

www.bmj.com

\begin{abstract}
Objectives To evaluate the associations in twins between within pair differences in birth weight and subsequent blood pressures as adults thereby removing the impact of potential parental confounding variables.

Design Historical cohort study.

Setting St Thomas's UK adult twin register, June 1992 to September 1995.

Participants 492 pairs of female twins (mean age 54 years).

Main outcome measures Mean within pair differences in adult blood pressure in each of four strata of within pair differences in birth weight $(0$, $1-500 \mathrm{~g}, 501-1000 \mathrm{~g},>1000 \mathrm{~g})$. Differences in blood pressure were analysed before and after adjustment for potential confounders between adult twins, after exclusion of those twin pairs including at least one twin taking antihypertensive drugs, and by zygosity. Results Reported mean birth weights of heavier and lighter twins were 2.51 (SD 0.61) v $2.12(0.59) \mathrm{kg}$ respectively. A graded inverse relation between strata of within pair differences in birth weight and differences in adult blood pressure was apparent, with an adjusted blood pressure range of $8.7 / 5.1 \mathrm{~mm} \mathrm{Hg}$ across the four strata (test for trend: systolic, $\mathrm{P}=0.05$; diastolic, $\mathrm{P}=0.09$ ). After excluding those women taking antihypertensive drugs the significance of the association was similar (systolic, $\mathrm{P}=0.04$; diastolic, $\mathrm{P}=0.10$ ). When differences in blood pressure were stratified for zygosity similar but non-significant trends were apparent.

Conclusion It would seem that birth weight is inversely associated with adult blood pressure and that this association is independent of parental confounding variables probably including, in view of the findings in monozygotic twins, genetic factors. The observed blood pressure differences are likely to result from retarded intrauterine growth due to placental dysfunction rather than inadequate maternal nutrition.
\end{abstract}

\section{Introduction}

A meta-analysis of 34 studies has shown a significant inverse relation between birth weight and subsequent levels of blood pressure. ${ }^{1}$ Compared with higher birthweight babies, low birthweight babies have been shown to have higher levels of blood pressure as children, ${ }^{2-4}$ adolescents, ${ }^{45}$ and adults, ${ }^{346-8}$ the association being more pronounced with age. ${ }^{16}$ It is hypothesised that some adverse aspect or aspects of intrauterine life, such as nutritional deficiencies at critical periods of fetal growth, programme the fetus to have higher levels of blood pressure after birth. ${ }^{9}$ The mechanisms invoked to produce this programming effect include the impact of impaired fetal growth on blood vessel growth ${ }^{8}$ or compliance $^{10}$ or the number of nephrons. ${ }^{11}$
Despite the consistency of the findings ${ }^{1}$ potential flaws in the body of evidence supporting the programming hypothesis have been perceived. ${ }^{12}$ These include the lack of evidence for increased adult mortality among twins, ${ }^{13}$ and the possibility that various parental factors-particularly maternal smoking - may confound the apparent relation between birth weight and blood pressure. $^{14}{ }^{15}$ Authors of a recent Swedish study concluded that the inverse association between birth weight and blood pressure was unlikely to be due to confounding by socioeconomic circumstances. ${ }^{16}$ However, another recent study showed that low birth weight was associated with higher blood pressure in later life in both the offspring and the mother. ${ }^{17}$ Furthermore, after correction for parental blood pressures, the apparent association between birth weight and subsequent blood pressure levels was attenuated and ceased to be significant. The authors concluded therefore that low birth weight was a feature of the inherited predisposition to hypertension and that parental blood pressure may be an important confounder of the apparent relation between birth weight and blood pressure.

By investigating the association within twin pairs between differences in birth weight and differences in their subsequent adult blood pressures, we avoided parental characteristics that potentially could confound the association between birth weight and blood pressure. Furthermore, by investigating differences in birth weight and differences in subsequent adult blood pressure in twin pairs the impact of parental genetic make up is also reduced, and among monozygotic twins is eliminated, as a potential confounder.

\section{Participants and methods}

\section{Participants}

Data were collected from unselected female twins who were recruited through a national media campaign as part of the St Thomas's UK adult twin register, which was initially set up as a cohort of female volunteers. ${ }^{18}$ Twins were invited to attend a central clinic where they underwent a physical examination and investigations including blood pressure measurement. Blood pressures were measured in each pair of twins at the same time by the same observer under the same conditions. Blood pressures were measured twice, 20 minutes apart, in the sitting position under standardised conditions with a standard mercury sphygmomanometer. The mean of these two readings was used in analyses. Information on medical history, drug use, lifestyle, and demographic variables was obtained by standardised nurse administered questionnaire. Questions on recalled birth weight (in kilograms or heavier or lighter status) and birth order were supplied two weeks before the interview, and interviewees were encouraged to consult their mothers for answers. Zygosity was assessed by standardised questionnaire, and DNA fingerprinting was used for confirmation. ${ }^{19}$ 
Between June 1992 and September 1995, when only females were included in the register, 641 pairs of twins were evaluated in the St Thomas's UK adult twin register study. Of these, 492 pairs $(76.8 \%)$ provided complete information on self reported birth weights, and valid blood pressure recordings were available for 479 of these.

\section{Differences in birth weight}

The reported difference in birth weight was calculated (in grams) between heavier and lighter twins. Reported differences were stratified into five categories determined a priori: 0, 1-500 g, 501-1000 g, 1001-1500 g, and greater than $1500 \mathrm{~g}$. As only 21 twin sets had birth weight differences greater than $1 \mathrm{~kg}$ the last two categories were combined in analyses.

\section{Smoking and alcohol consumption}

Smoking was classified as never, current, or past smoker. Current smokers were defined as those participants who had smoked at least one cigarette per day in the past month. Current alcohol consumption was classified into one of seven categories: never, occasionally but less than one unit per week, and 1-5, 6-10, 11-15, 16-20, and more than 20 units per week. Differences in continuous variables between twin pairs were calculated in the same way as for birthweight differences (heavier minus lighter). For twin pairs with the same birth weight a random order for calculating differences was allocated. For potentially confounding categorical variables, the within pair differences were included in the regression model as a series of indicator variables representing the difference within each pair.

\section{Potential confounders and statistical methods}

Weight, height, smoking habit, and alcohol consumption were considered as potential confounders. Of 479 twin pairs with reported birth weight and valid blood pressure levels, 406 had recorded data on all these variables. Of these 406 twin pairs, 167 were monozygotic, 237 were dizygotic, and two were unclassified. Mean blood pressures and mean within pair differences in blood pressure are presented both unadjusted and after adjustment for the four potential confounders. Correlations between within pair differences in adult blood pressure and within pair differences in birth weight were examined with and without adjustment for potential confounders, but only partial (adjusted) correlation coefficients are presented. The impact of antihypertensive drugs on results was evaluated by carrying out analyses after excluding all twin pairs in which at least one twin was taking such drugs and alternatively by adjusting for blood pressure treatment.

\section{Results}

Table 1 shows the various characteristics of the twins (mean age 54 years) by self reported birthweight status.
Table 1 Characteristics of those participants with known blood pressure level by self reported birthweight status. Values are numbers (percentages) unless stated otherwise

\begin{tabular}{|c|c|c|c|}
\hline \multirow[b]{2}{*}{ Characteristic } & \multicolumn{3}{|c|}{ Birth weight } \\
\hline & $\begin{array}{l}\text { Heavier } \\
(\mathrm{n}=399)\end{array}$ & Lighter ( $\mathrm{n=399)}$ & Same $(n=160)$ \\
\hline Mean (SD) birth weight $(\mathrm{kg})$ & $2.5(0.6)$ & $2.1(0.6)$ & $2.4(0.6)$ \\
\hline Mean (SD) age (years) & $53.7(8.7)$ & $53.7(8.7)$ & $56.4(7.5)$ \\
\hline Mean (SD) height $(\mathrm{cm})^{\star}$ & $162.7(5.9)$ & $161.7(5.9)$ & $161.2(5.7)$ \\
\hline Mean (SD) weight $(\mathrm{kg}) \dagger$ & $64.4(10.4)$ & $63.9(10.5)$ & $63.0(9.2)$ \\
\hline Mean (SD) shared home until age (years) $\ddagger$ & $20.2(5.8)$ & $20.2(5.8)$ & $20.2(3.0)$ \\
\hline Current antihypertensive treatment & $59(15)$ & $58(15)$ & $24(15)$ \\
\hline \multicolumn{4}{|l|}{ Smoking status: } \\
\hline Current & $59(15)$ & $71(18)$ & $22(14)$ \\
\hline Past & $119(30)$ & $108(27)$ & $50(31)$ \\
\hline \multicolumn{4}{|l|}{ Current alcohol consumption§: } \\
\hline Never & $38(10)$ & $39(11)$ & $12(9)$ \\
\hline Occasionally & $121(33)$ & $120(32)$ & $58(45)$ \\
\hline $1-5$ units/week & $108(29)$ & $102(28)$ & $28(22)$ \\
\hline 6-10 units/week & $65(18)$ & $68(18)$ & $19(15)$ \\
\hline$>11$ units/week & $38(10)$ & $41(11)$ & $13(10)$ \\
\hline
\end{tabular}

${ }^{*} n=387$ in heavier group; $n=386$ in lighter group; and $n=157$ in same group. $+n=384$ in heavier group; $n=386$ in lighter group; and $n=156$ in same group. $\ddagger n=396$ in heavier group; $n=396$ in lighter group; and $n=160$ in same group. $\S n=370$ in heavier group; $n=370$ in lighter group; and $n=130$ in same group.

Mean levels of systolic and diastolic blood pressure before and after adjustment for potential confounders were higher among the lighter than the heavier group of twins (table 2 and website). Adjusted within pair mean differences in blood pressure (heavier minus lighter $)$ were $-4.6 /-3.0 \mathrm{~mm} \mathrm{Hg} \quad(\mathrm{P}=0.07$ and $\mathrm{P}=0.04$ respectively), these differences increasing to $-6.8 /-4.1 \mathrm{~mm} \mathrm{Hg}(\mathrm{P}=0.02$ and $\mathrm{P}=0.02$ respectively) after excluding those pairs in which one or both twins were taking antihypertensive drugs.

Within pair differences in birth weight between twins and differences in adult blood pressure levels adjusted for confounders were not significantly correlated (systolic blood pressure: $r=-0.08, \mathrm{P}=0.12$; diastolic blood pressure $r=-0.06, \mathrm{P}=0.22$ ). However when twin pairs, which included at least one twin taking antihypertensive drugs, were omitted from analyses, stronger associations became apparent (systolic: $r=-0.14, \mathrm{P}=0.01$; diastolic: $r=-0.12$ : $\mathrm{P}=0.04$ ). Furthermore, when twin pairs were stratified into one of four levels of birthweight differences, including and excluding any participants taking antihypertensive drugs, an inverse relation between birth weight and adult blood pressure was apparent. This association was of borderline significance for systolic blood pressure when all twin pairs were included in analyses, and the significance of the association was marginally increased for systolic blood pressure when those receiving treatment were excluded (table 3 and website).

When blood pressure differences were stratified by zygosity (table 3 and website) similar trends were apparent although less robust for diastolic pressure among monozygotic twins, and no trends were statisti-

Table 2 Unadjusted and adjusted adult blood pressure levels by self reported birthweight status. Values are mean (SEM)

\begin{tabular}{|c|c|c|c|c|c|c|}
\hline \multirow{2}{*}{$\begin{array}{l}\text { Blood pressure } \\
(\mathrm{mm} \mathrm{Hg})\end{array}$} & \multicolumn{3}{|c|}{ Unadjusted } & \multicolumn{3}{|c|}{ Adjusted* } \\
\hline & Heavier $(n=345)$ & Lighter $(n=345)$ & Same $(n=122)$ & Heavier $(n=345)$ & Lighter $(n=345)$ & Same $(n=122)$ \\
\hline Systolic & $131.5(1.1)$ & $133.4(1.1)$ & $132.4(1.9)$ & $132.8(1.4)$ & $134.6(1.4)$ & $131.3(2.0)$ \\
\hline Diastolic & F80.7 (0.6) & $81.6(0.6)$ & $80.9(1.0)$ & $80.0(0.8)$ & $81.9(0.8)$ & $79.5(1.1)$ \\
\hline
\end{tabular}

${ }^{*}$ Adjusted for age, height, weight, smoking status, and current alcohol consumption. 
Table 3 Unadjusted and adjusted within pair differences in adult blood pressure levels by differences in self reported birth weights in all twins, in those who currently did not receive antihypertensive treatment, and by zygosity. Values are mean (SEM)

Difference in blood pressure*

\begin{tabular}{|c|c|c|c|c|c|}
\hline \multirow{3}{*}{$\begin{array}{l}\text { Difference in birth } \\
\text { weight }(g)\end{array}$} & \multirow[b]{3}{*}{ No } & \multirow{2}{*}{\multicolumn{2}{|c|}{ Systolic (mm Hg) }} & \multirow{2}{*}{\multicolumn{2}{|c|}{ Diastolic $(\mathrm{mm} \mathrm{Hg})$}} \\
\hline & & & & & \\
\hline & & Unadjusted & Adjusted† & Unadjusted & Adjusted† \\
\hline \multicolumn{6}{|l|}{ All twins } \\
\hline 0 & 61 & $2.38(2.78)$ & $-0.61(3.59)$ & $0.98(1.63)$ & $-1.18(2.09)$ \\
\hline$\leqslant 500$ & 273 & $-0.99(1.33)$ & $-2.79(2.50)$ & $-0.55(0.77)$ & $-2.13(1.46)$ \\
\hline $501-1000$ & 51 & $-5.00(3.04)$ & $-6.87(3.61)$ & $-2.16(1.79)$ & $-3.71(2.10)$ \\
\hline$>1000$ & 21 & $-6.57(4.74)$ & $-9.28(5.50)$ & $-3.81(2.79)$ & $-6.28(3.00)$ \\
\hline Trend & & $P=0.04$ & $P=0.05$ & $P=0.08$ & $P=0.09$ \\
\hline \multicolumn{6}{|c|}{ Twins currently not receiving antihypertensive treatment } \\
\hline 0 & 44 & $-0.23(3.11)$ & $-4.53(4.03)$ & $-0.11(1.92)$ & $-2.71(2.48)$ \\
\hline$\leqslant 500$ & 275 & $-2.37(1.44)$ & $-5.30(2.91)$ & $-1.10(0.89)$ & $-3.15(1.79)$ \\
\hline $501-1000$ & 38 & $-5.13(3.35)$ & $-9.54(4.10)$ & $-3.16(2.07)$ & $-6.05(2.52)$ \\
\hline$>1000$ & 17 & $-12.2(5.01)$ & $-16.1(5.52)$ & $-5.59(3.09)$ & $-8.84(3.39)$ \\
\hline Trend & & $P=0.04$ & $P=0.04$ & $P=0.09$ & $P=0.10$ \\
\hline \multicolumn{6}{|l|}{ Monozygotic twins } \\
\hline 0 & 27 & $4.26(4.00)$ & $4.09(6.21)$ & $1.85(2.46)$ & $-1.69(3.74)$ \\
\hline$\leqslant 500$ & 116 & $0.53(1.94)$ & $-0.004(5.09)$ & $-0.36(1.18)$ & $-3.81(3.06)$ \\
\hline $501-1000$ & 17 & $-4.41(5.04)$ & $-4.38(6.43)$ & $2.06(3.10)$ & $0.55(3.87)$ \\
\hline$>1000$ & 7 & $-6.86(7.85)$ & $-6.32(9.72)$ & $-2.14(4.83)$ & $-6.38(5.61)$ \\
\hline Trend & & $P=0.10$ & $P=0.13$ & $P=0.67$ & $P=0.82$ \\
\hline \multicolumn{6}{|l|}{ Dizygotic twins } \\
\hline$\overline{0}$ & 34 & $0.88(3.85)$ & $-2.64(4.68)$ & $0.29(2.20)$ & $-1.27(2.68)$ \\
\hline$\leqslant 500$ & 155 & $-2.40(1.83)$ & $-4.60(3.03)$ & $-0.75(1.04)$ & $-1.85(1.74)$ \\
\hline $501-1000$ & 34 & $-5.29(5.85)$ & $-7.22(4.51)$ & $-4.26(2.20)$ & $-5.69(2.58)$ \\
\hline$>1000$ & 14 & $-6.43(6.01)$ & $-9.39(6.40)$ & $-4.64(3.42)$ & $-6.28(3.66)$ \\
\hline Trend & & $P=0.20$ & $P=0.27$ & $P=0.08$ & $P=0.08$ \\
\hline
\end{tabular}

*Heavier minus lighter.

†Adjusted for differences in weight, height, smoking status, and current alcohol consumption.

cally significant. Further adjustment for blood pressure treatment did not significantly affect these findings, and similar trends were apparent when those who were taking antihypertensive drugs were excluded from the analyses (data not shown).

\section{Discussion}

These data suggest that the observation previously made in over 30 studies, ${ }^{1}$ that birth weight is inversely associated with blood pressure levels later in life, is not due to parental confounding factors such as maternal smoking. The mean differences in adjusted blood pressure between adult twins associated with a difference in birth weight between twins of more than $1 \mathrm{~kg}$ (average difference $1506 \mathrm{~g}$ ) compared with those twins with no reported birth weight differences was $8.7 / 5.1 \mathrm{~mm} \mathrm{Hg}$ (table 3 and website), which is equivalent to $5.8 / 3.4 \mathrm{~mm}$ $\mathrm{Hg}$ per $\mathrm{kg}$ difference in birth weight. This size of difference is larger than that observed in most previous studies of unrelated adults ${ }^{1}$ and may reflect the impact in these studies of various confounding characteristics due to the more diverse lifestyles of unrelated adults or to having different parental influences that could have obscured the true size of the association. Given these findings and the fact that twins are on average smaller than singletons, the prevalence of hypertension might be expected to be higher among twins than singletons. To our knowledge, no published data are available to support this expectation, indeed a recent study reported lower systolic blood pressures among twins compared with singletons at age 9 and 18 years. ${ }^{20}$ Interestingly, the treatment rates for hypertension shown in table 1 are marginally higher than those reported in a recent nationally representative sample of women of a similar age. ${ }^{21}$

When twin pairs were stratified by zygosity similar patterns in blood pressure differences with birthweight differences were observed but were not significant. The trend was less clear for diastolic blood pressure among monozygotic twins. However, all these findings should be considered in the light of the small numbers in some weight difference strata and the fact that other determinants of adult blood pressures (for example, electrolyte intakes) could not be considered in analyses. Furthermore because birth weights were self reported they were likely to be variably inaccurate, although the recall of twins is likely to be superior to singletons and this method is considered to be acceptably valid in the population setting. ${ }^{22-24}$

The interpretation of blood pressure levels of those taking antihypertensive drugs is difficult because the real blood pressure differences within twin pairs is likely to be reduced or even reversed if one twin receives blood pressure lowering therapy. Data could have been modified to compensate for this problem by adjusting for blood pressure treatment in the analyses, or by omitting those twin pairs that included at least one twin taking blood pressure drugs and thereby removing potential obfuscation of the real blood pressure difference. An alternative approach was to allocate some arbitrary blood pressure level above the median to those receiving treatment ${ }^{25}$ although this method would not necessarily compensate for the problem at the individual twin pair level. Overall, adjustment for blood pressure treatment had little if any effect on the findings shown, although the exclusion of those taking 
antihypertensive drugs did seem to enhance the apparent association (table 3 and website).

Whether to adjust for adult anthropometric variables-particularly body mass index-has been debated in the context of earlier studies. ${ }^{7}{ }^{12}$ However, as expected from finding similar mean adult levels among twins who reported heavier, lighter, or the same birth weight (table 1), the inclusion of adjustment for height, weight, or body mass index in the analyses did not influence the results importantly. The inverse association between birth weight and adult blood pressure may have occurred by chance, bias, or confounding, or because the two variables are causally related. The strength and consistency of the association makes chance an unlikely explanation. ${ }^{1}$ The reproducibility of the findings in over 30 different studies of many different types of population using different methods makes some systematic error in study design or conduct also unlikely. The most frequently cited criticisms relating to the programming hypotheses have been on the basis of the likelihood of some form of confounding inducing low birth weight and also increasing subsequent blood pressure levels.

The data presented in our study eliminate the likelihood of parental confounders inducing the sort of association between blood pressure and birth weight previously reported although, as suggested by one study ${ }^{17}$ confounding may have contributed to some of the observed associations. The findings in monozygotic twins, although not statistically significant, suggest that the previously reported effects of low birth weight on subsequent adult blood pressure levels are also not due to genetic confounding-that is, not the result of a gene causing small birth weight and subsequently raised blood pressure levels.

Hence on the basis of this study, it seems reasonable to exclude confounding by genetic or environmental factors as the single explanation for the inverse association between birth weight and blood pressure. Moreover, given that chance and bias seem to be equally unlikely explanations, it seems that some aspect of intrauterine life that limits birth weight is independently and causally associated with subsequent higher blood pressure levels.

It has been proposed that retarded intrauterine growth due to inadequate fetal nutrition causes raised blood pressure levels in adulthood. ${ }^{9}$ Our study cannot identify which of two likely candidates-placental dysfunction or inadequate maternal nutrition-is responsible for the inadequate fetal nutrition. However, in our study heavier and lighter twins were exposed to the same maternal diet and hence, although not excluding the role of inadequate maternal nutrition in other studies, is more compatible with the hypothesis that placental dysfunction is a cause of the retarded intrauterine growth, which is linked with high adult blood pressure.

We thank Sandy Johnson for assistance with the preparation of the manuscript, Ursula Perks and other research interviewers for administering the questionnaires, and all the twins who volunteered their time.

Contributors: NRP instigated this investigation, advised on analyses, and wrote the initial draft of the manuscript. CLC compiled the dataset, performed all analyses, and commented on relevant parts of the manuscript. TDS began the twins register and along with AJM and HS was responsible for the original data collection, advised on analyses, and contributed to and
- Among adult twins, blood pressures tend to be lower among those twins who were heavier at birth

- Strata of within pair birthweight differences of twins show a graded inverse relation with adult blood pressure differences

- Monozygotic and dizygotic twins show a similar inverse association between birthweight differences and adult blood pressure differences

- The inverse association between birth weight and adult blood pressure is independent of parental confounding variables

reviewed all sections of the manuscript. NRP and TDS will act as guarantors for the paper.

Funding: The St Thomas's UK adult twin register is part funded by grants from the Wellcome Trust, Medical Research Council, Arthritis and Rheumatism Campaign, Chronic Disease Research Foundation, British Heart Foundation, and Gemini Research.

Competing interests: None declared.

1 Law CM, Shiell AW. Is blood pressure related to birth weight? The strength of evidence from a systematic review of the literature.J Hyperten 1996;14:935-41.

2 Hashimoto N, Kawaaki T, Kikuchi T, Takahashi H, Uchiyama M. The relationship between the intrauterine environment and blood pressure in 3-year-old Japanese children. Acta Paediatr 1996:85:132-8.

3 Vancheri F, Alletto M, Burgio A, Fulco G, Paradiso R, Piangiamore M. Relazione inversa tra crescita fetale e pressione arteriosa in eta infantile $\mathrm{e}$ adulta. G Ital Cardiol 1995;25:833-41.

4 Zureik M, Bonithon-Kopp C, Lecomte E, Siet G, Ducimetiere P. Weights at birth and in early infancy, systolic pressures and left ventricular structure in subjects aged 8 to 24 years. Hypertension 1996;27:339-45.

5 Macintyre S, Watt G, West P, Ecob R. Correlates of blood pressure in 15 year olds in the West of Scotland. J Epid Comm Health 1991;45:143-7.

6 Law CM, de Swiet M, Osmond C, Fayers PM, Barker DJP, Cruddas AM, et al. Initiation of hypertension in utero and its amplification throughout life. BMJ 1993;306:24-7.

7 Leon DA, Koupiloa I, Lithell HO, Berglund L, Mohsen R, Vagero D, et al. Failure to realise growth potential in utero and adult obesity in relation to blood pressure in 50 year old Swedish men. BMJ 1996;312:401-6.

8 Martyn CN, Barker DJP, Jesperen S, Greenwald S, Osmond C, Berry C. Mrowth in utero, adult blood pressure, and arterial compliance. Br Heart J 1995; 73:116-21.

9 Barker DJP. Mothers, babies and disease in later life. London: BMJ Publising, 1994.

10 Law CM, Barker DJP. Fetal influences on blood pressure. J Hypertens 1991;303:1019-22.

11 Brenner BM, Chertow GM. Congenital oligonephropathy and the etiology of adult hypertension and progressive renal injury. Am J Kidney Dis 1994;23:171-5

12 Paneth N, Susser M. Early origins of coronary heart disease (the Barker hypothesis). BMJ 1995;310:411-2.

13 Christensen K, Vaupel J, Holm NV, Yashin AI. Twin mortality after age six: fetal origin hypothesis versus twin method. BMJ 1995;310:432-6.

14 Matthes JWA, Lewis PA, Davies DP, Bethel JA. Relation between birth weight at term and systolic blood pressure in adolescence. BMJ 1994:308:1074-7.

15 Ben-Shlomo Y, Davey Smith G. Deprivation in infancy or adult life; which is more important for mortality risk? Lancet 1991;337:530-4.

16 Koupilova I, Leon DA, Vagero D. Can counfounding by sociodemographic and behavioural factors explain the association between size at birth and blood pressure at age 50 in Sweden? J Epid Comm Health 1997;51:14-8.

17 Walker BR, McConnachie A, Noon JP, Webb DJ, Watt GCM. Contribution of parental blood pressures to association between low birth weight and adult high blood pressure: cross sectional study. BMJ 1998;316:834-7.

18 Boomsma DI. Twin registers in Europe: an overview. Twin Res 1998;1 34-51.

19 Spector TD, Cicuttini F, Baker J, Loughlin J, Hart D. Genetic influences on osteoarthritis in women: a twin study. BMJ 1996;312:940-4.

20 Williams S, Poulton R. Twins and maternal smoking: ordeals for the fetal origins hypothesis? A cohort study. BMJ 1999;318:897-900.

21 Colhoun HM, Dong W, Poulter NR. Blood pressure screening, management and control in England; results from the health survey for England 1994. J Hypertens 1998;16:747-53.

22 Aelsson G, Rylander R. Validation of questionnaire reported miscarriage malformation and birthweight. Int J Epidemiol 1984;13:94-8.

23 Little R. Birthweight and gestational age: mothers' estimates compared with state and hospital records. Am J Public Health 1986;76:1350-1.

24 Troy LM, Michels KB, Hunter DJ, Spiegelman D, Manson JE, Colditz GA et al. Self-reported birthweight and history of having been breastfed among younger women: an assessment of validity. Int J Epidemiol 1998;25:122-7.

25 White I, Chaturvedi N, McKeigue P. Median analysis of blood pressure for a sample including treated hypertensives. Stat Med 1994;13:1635-41.

(Accepted 21 July 1999) 